\title{
Uso de las redes sociales y rendimiento académico de estudiantes de preparatoria de la Universidad Autónoma de Sinaloa
}

Rosalba Trinidad Chávez-Moreno

rochamor2@gmail.com

Universidad Autónoma de Occidente

Francisco Antonio Romero-Leyva

roleyva131@hotmail.com

Universidad Autónoma Indígena de México

Teodoro Lugo-Tapia

lugot63@hotmail.com

Universidad Autónoma de Occidente

Carlos Alberto Apodaca-López

carlosp175@live.com.mx

Universidad Autónoma de Occidente

Georgina Félix-Ortiz

gfelixortiz@gmail.com

Universidad Autónoma de Occidente

Silvia Ortiz- Castro

Silvia.ortiz@uadeo.mx

Universidad Autónoma de Occidente

\section{RESUMEN}

Es sorprendente la forma en que los estudiantes del nivel medio superior aprenden a manejar las nuevas tecnologías de la información y la destreza que tienen para navegar en el mundo del internet, ingresando a las páginas web que estén a disposición y que llamen la atención de los jóvenes; cuando tienen horas libres, acceden no precisamente para cumplir con tareas escolares, sino a buscar cualquier clase de diversión, principalmente el uso de redes sociales donde las principales actividades que realizan lo es el chat, compartir fotografías y videos que es de nula relevancia para el proceso de aprendizaje escolar de los estudiantes nivel bachillerato pertenecientes a la preparatoria de la UAS El Fuerte.

La presente investigación denominada "Uso de las redes sociales y rendimiento académico de estudiantes de preparatoria de la universidad autónoma de sinaloa, 2020" es una investigación que tiene como objetivo principal determinar la influencia que ejerce el uso de las redes sociales de internet en el rendimiento académico de los estudiantes de 
la Unidad Académica Preparatoria UAS de El Fuerte, Sinaloa, para lo cual se utilizó una metodología cuantitativa que de acuerdo al diseño de la investigación no experimental transaccional y correlacional en cuanto a su alcance, en el que se estudia la relación de las variantes de investigación uso de las redes sociales y rendimiento académico en alumnos con promedio igual o menor a 7.0 en la que de acuerdo a la hipótesis se plantea que el uso de las redes sociales en internet, afecta significativamente el rendimiento académico de los estudiantes que fueron objeto de la investigación, los cuales fueron 137 alumnos con estas características de promedio escolar, donde principalmente se utilizó como instrumento o medio de obtención de datos la observación participante del autor y la encuesta, la cual fue procesada con el programa SPSS stadistic versión v20 con el que se estableció una relación de resultados en tablas de contingencia que permitió hacer un análisis cuantitativo con una interpretación cualitativa de los resultados obtenidos.

Palabras claves: redes sociales; rendimiento académico; internet; estudiantes; comunicación; educación.

Artículo recibido: 02 Setiembre. 2021 Aceptado para publicación: 30 Setiembre. 2021

Correspondencia: rochamor2@gmail.com Conflictos de Interés: Ninguna que declarar 


\title{
Use of social media and academic performance of high school students of the Universidad Autónoma de Sinaloa
}

\begin{abstract}
Is amazing how students of the high school Universidad Autónoma de Sinaloa from El Fuerte, learn and use the information and communication technologies (ICT) even how they have the hability to surf the Internet with the purpose to enter in web sites or social networks that are available for all the people around the world. When students have free time they prefer to access the internet not for doing homeworks or any academic school projects, they do this just to have fun in the social networks where they can send texts to other people, share pictures, watch videos and down load or up load all type of information. This activity done for the students is irrelevant in the teaching and learning process of the students of the high school Universidad Autónoma de Sinaloa (UAS) campus El Fuerte.
\end{abstract}

This research denominated. "Use of the social networks and academic performance of the students of the universidad autónoma de sinaloa campus el fuerte, 2020" is focused on determining the influence of the social networks in the academic performance of the students of the Universidad Autónoma de Sinaloa Campus El Fuerte. This research was done using a quantitative methodology and According to the design of the research nonexperimental transactional and correlational in terms of its scope, which studies the relationship between these variants: The use of social networks and academic performance in students with an average of 7.0 or less. According to the hypothesis says that the use of social networks on the Internet affects the academic performance of the 137 students who were the object of the research, students with the school average of 7.0 or less. Also it was used as an instrument to obtain data, the direct observation and the survey, information that was processed with the statistical program SPSS version v20 with which a relation of results was established in contingency tables that allowed a quantitative analysis with a qualitative interpretation of the results obtained.

Keywords: social networks; academic performance; internet; students; communication; education. 


\section{INTRODUCIÓN}

Estamos inmersos en una sociedad nueva, llamada sociedad del conocimiento, la cual nos demanda altos conocimientos acerca de las Tecnologías de la Información y Comunicación (TIC), para poder sobrevivir en ella.

Son pocas las personas que se encuentran alejadas de las TIC, pues se requiere de ellas para muchas de las actividades diarias en el hogar, en empresas y por supuesto en instituciones educativas. Al hacer uso de estas herramientas, los adolescentes y jóvenes son capaces de desafiar los retos que se presentan en su entorno académico.

Para todo tipo de aplicaciones educativas, las Tecnologías de la Información y Comunicación (TIC) son medios y no fines. Es decir, son herramientas y materiales de construcción que facilitan el aprendizaje, el desarrollo de habilidades y distintas formas de aprender, estilos y ritmos de los aprendices. Del mismo modo, la tecnología es utilizada tanto para acercar al aprendiz al mundo, como el mundo al aprendiz.

El desarrollo acelerado de la sociedad de la información está suponiendo retos impensables desde hace unos años, para la educación y el proceso de enseñanza aprendizaje.

Todo ello es producto de la globalización del desarrollo de las tecnologías y la comunicación, donde internet juega el papel rector y específicamente las redes sociales y que según la amplitud del concepto de red social de internet considero que es un espacio virtual de comunicación entre personas con algún rasgo en común en la que sus usuarios pueden contactar con familiares, amigos o desconocidos, y compartir contenidos sin que importe el tiempo, el espacio o la ubicación. Por lo tanto, sería un sistema abierto de interacción social, en permanente construcción gracias a las continuas aportaciones de cada usuario.

Este producto tecnológico se ha generalizado de tal manera que ya ocupa la totalidad de los países, regiones y la gran mayoría de la población. Siendo muy preocupante el uso que le dan los niños, adolescentes, jóvenes e incluso adultos a las redes sociales, llegando a ocupar la totalidad de su tiempo, convirtiéndose en una conducta adictiva a las mismas. Por supuesto que trae consecuencias negativas en la formación sana y desarrolladora del hombre, por ello es importante estudiar cómo afecta las redes sociales de internet en el rendimiento académico de los estudiantes. 
En este el uso del tiempo libre en cuanto acción educativa implica la búsqueda del perfeccionamiento, lo mejor de la persona y por extensión, de la comunidad. Esta acción debe hacerse realidad a través de la actividad mental y física, y en la medida que se conjuguen, proporcionarán una situación formativa óptima. Un segundo aspecto dentro de ese proceso sería la intencionalidad que se relaciona con la motivación y el fin que persigue una intervención determinada y un tercer aspecto sería la sistematización en la proyección temporal y metodología de la experiencia para profundizar en la acción educativa y su trascendencia.

El nivel medio superior o educación preparatoria, tiene como objetivo el de formar estudiantes con las bases necesarias para enfrentar los retos y desafíos característicos de su etapa siguiente como es la licenciatura, así como adoptar un papel orientador para ayudar a alumno a elegir cuál es su vocación profesional y el rumbo académico que continuará en los subsecuentes años.

Por ello, se concibe esta investigación, que está encaminada a determinar la repercusión del uso de las redes sociales en el rendimiento académico de los estudiantes de preparatoria, por ello contribuirá a un conocimiento más profundo de la relación de ambos factores, en nuestros alumnos, y cómo actuar al respecto.

El trabajo se desarrolló en la Unidad Académica Preparatoria UAS El Fuerte; esta institución en una zona rural donde los estudiantes cuentan con pocos espacios recreativos sanos, por ello la situación se agudiza.

Se ha seleccionado esta población, por el conocimiento del autor de las características de la muestra, por las posibilidades de acceder a ella y por la vulnerabilidad de la misma.

\section{MÉTODOS Y TÉCNICAS DE INVESTIGACIÓN}

Para la presente investigación se utilizó el enfoque cuantitativo, "usa la recolección de datos para probar hipótesis con la base en la medición numérica y el análisis estadístico para establecer patrones de comportamiento de una población".

El enfoque cuantitativo representa, un conjunto de procesos, es secuencial y probatorio. Cada etapa precede a la siguiente y no podemos "brincar" o eludir pasos. El orden es riguroso, aunque desde luego, podemos redefinir alguna fase. Parte de una idea que va acotándose y, una vez delimitada, se derivan objetivos y preguntas de investigación, se revisa la literatura y se construye un marco o una perspectiva teórica. De las preguntas se establecen hipótesis y determinan variables; se traza un plan para probarlas (diseño); se 
miden las variables en un determinado contexto; se analizan las mediciones obtenidas utilizando métodos estadísticos, y se extrae una serie de conclusiones respecto de la o las hipótesis. (Sampieri, 2014)

El trabajo es de carácter analítico, las variables de estudio fueron las redes sociales y el rendimiento académico. Para poder realizar este análisis fue necesario que las unidades pudieran ser observadas y caracterizadas durante el proceso de la investigación empírica. Después se determinó qué unidades serían las que aportarán la evidencia empírica para responderlas preguntas y contrastar las hipótesis planteadas.

Para esta tarea fue clave en el pasaje del marco conceptual al marco teórico por ello se sumó la tarea de identificar y demarcar, con máxima precisión, qué unidades se iban a analizar empíricamente.

Posteriormente se definieron las propiedades observadas (las variables), estas fueron asignadas a unidades con relación a las cuales se contrastaron la hipótesis y se sacaron conclusiones, en el proceso de la investigación se focalizo en aprender el significado que los participantes otorgan al problema o fenómeno en cuestión, no en el significado que como investigador se le asigna tampoco a lo que expresa la literatura al respecto.

Como primera fase se realizó una recopilación bibliográfica, por lo que se dio una minuciosa revisión de bibliografía en relación a la temática, que consistió en reunir toda la información que fuera la base importante del objeto de estudio de la investigación, además, se realizó una consulta en relación con los resultados de las calificaciones del sistema de la Dirección de escolar de la Unidad Académica Preparatoria El Fuerte, de la Universidad Autónoma de Sinaloa, con el fin de contar con la base de datos de los promedio de los alumnos, que posteriormente serian tomados como muestra para la aplicación de una encuesta, así como también investigaciones en relación a las redes sociales y el rendimiento académico con el objetivo de realizar análisis comparativo de las variables de estudio y dar sustento al presente proyecto, documentando con artículos científicos, otros estudios, libros de textos, páginas web, entre otros.

La observación participante según (Malinowski, 1967) “es el eje vertebrador de la propuesta etnográfica enmarcada en una conceptualización acerca de la cultura como sistema".

En la segunda fase que consistió en el trabajo de campo; recogida de información por medio de la aplicación de encuestas dirigidas a alumnos con promedio menor o igual a 
7.0 de acuerdo al sistema de escolar con el fin de indagar, explorar y conocer las diferentes dimensiones de la temática, a los encuestados se explicó el propósito de la investigación. Por medio de la observación participante, se registraron evidencias principalmente del uso que el alumno da a las redes sociales, con qué fin lo hace, de qué tipo de red social es miembro, con qué frecuencia el alumno accede a las redes sociales y el tiempo que le destina de acuerdo a su uso, además de que el alumno no destina el uso de redes sociales o acceso a internet en la escuela o en alguna otra parte para actividades académicas y que este posterga actividades escolares para dedicarle más tiempo a el entretenimiento, amistades y relaciones sentimentales.

Fundamentalmente, la observación participante es la integración del observador en el espacio de la comunidad observada; su principal uso, menciona Callejo (2002) se encuentra en el estudio de lo que relativamente se sale de la norma: lo que todavía no se entiende, las otras culturas, los grupos semicultos o clandestinos y lo que tiende a encerrarse entre los muros de las instituciones (instituciones totales, centros laborales y laboratorios). (Callejo Gallego, 2002)

Se contempló que la observación comprendiera tres actividades: las observaciones mismas, la toma de notas y la interpretación.

Por lo que se realizó el análisis de la información obtenida en el proceso de investigación, lo cual se sustenta en cuatro apartados fundamentales: a) revisión bibliográfica); b) observación participante, c) trabajo de campo y d) revisión de documentos institucionales. Finalmente en la última fase; Se realizó un análisis intensivo de la información, caracterizado por la reducción de los datos, la disposición de los mismos y la obtención de resultado, lo que además de contribuir a una mayor comprensión de la problemática y a la confirmación de las interrogantes presentadas, favoreció el planteamiento de algunos elementos teóricos, como resultado de esta indagación.

El proceso de validación del estudio se realizó a través de la comparación de los resultados de las diferentes técnicas de recolección de datos y análisis.

\section{Operación de las variables}

Para llevar a cabo la etapa investigativa se procede a operacionalizar las variables; Redes Sociales y Rendimiento Académico, con el fin de identificar las causas que hacen que las redes sociales de internet sean el mayor interés para los estudiantes, y así mismo las ventajas y desventajas que de esto se derivan. 
Variable Independiente: Redes Sociales Para la presente investigación, el investigador propone la siguiente definición: Una Red Social actualmente es considerada como un espacio virtual en donde convergen grupos de individuos, para interactuar en contextos o situaciones que reflejen un interés común, facilitando la comunicación inmediata, ágil y rápida, de los participantes en el proceso comunicativo.

\section{Dimensiones: Variable Independiente}

- Intereses Comunes (IC)

- $\quad$ Tipo de Información Compartida (TIC)

- $\quad$ Beneficios de Comunicación (BC)

- Comunicación Virtual (CV)

Tabla 1. Indicadores: Variable independiente

\begin{tabular}{|c|c|}
\hline Dimensiones & Indicadores \\
\hline Intereses Comunes (IC) & $\begin{array}{l}\text { 1. Amistad } \\
\text { 2. Relaciones Personales } \\
\text { 3. Compañeros de Estudio }\end{array}$ \\
\hline Tipo de Información Compartida (TIC) & $\begin{array}{l}\text { 4. Fotografías y videos } \\
\text { 5. Sentimientos y Emociones } \\
\text { 6. Académica }\end{array}$ \\
\hline Beneficios de Comunicación (BC) & $\begin{array}{l}\text { 7. Comunicación rápida con los contactos. } \\
\text { 8. Intercambio de ideas } \\
\text { 9. Publicación de actividades estudiantiles. } \\
\text { 10. Entretenimiento (juegos) }\end{array}$ \\
\hline Comunicación Virtual (CV) & $\begin{array}{l}\text { 11. Poseer cuenta en una red social. } \\
\text { 12. No poseer cuenta en una red social. } \\
\text { 13. Utilización de la red social entre } 1 \text { a } 3 \text { horas al } \\
\text { día. } \\
\text { 14. Utilización de la red social } 5 \text { o más horas al día. }\end{array}$ \\
\hline
\end{tabular}

Tabla 2. Escala para establecer la finalidad de las redes sociales

\begin{tabular}{|l|c|}
\hline \multicolumn{1}{|c|}{ Finalidad de las Redes Sociales } & Indicadores \\
\hline Encontrar Amistades & $1,4,7,8$ \\
\hline Establecer relaciones personales & $2,4,5,8$ \\
\hline Compartir Información Académica & $3,6,8,9$ \\
\hline Entretenimiento y ocio. & $1,4,10$ \\
\hline
\end{tabular}

Tabla 3. Escala para establecer el uso de las redes sociales

\begin{tabular}{|l|c|}
\hline \multicolumn{1}{|c|}{ Uso de las Redes Sociales } & Indicadores \\
\hline Excesivo & 10,14 \\
\hline Moderado & $10,12,13$ \\
\hline No se manifiesta & 11 \\
\hline
\end{tabular}




\section{Variable Dependiente: Rendimiento Académico}

Para la presente investigación, el investigador propone la siguiente definición: El rendimiento académico, es la forma en que se miden, los conocimientos, las capacidades y habilidades que desarrolla el estudiante en su proceso de aprendizaje, que le permite la construcción de sus propios conocimientos y le sean útiles en la resolución de las problemáticas a las cuales se enfrentara en su vida como estudiante y profesionista, las cuales se verán reflejadas en su desarrollo personal y social.

\section{Dimensiones: Variable Dependiente}

- Capacidades y Habilidades $(\mathrm{CH})$

- Proceso de aprendizaje (PZ)

- Crecimiento personal y social (CPS)

\section{Indicadores: Variables dependientes}

Tabla 4. Indicadores para la variable dependiente de rendimiento académico

\begin{tabular}{|l|l|}
\hline \multicolumn{1}{|c|}{ Dimensiones } & \multicolumn{1}{c|}{ Indicadores } \\
\hline Capacidades y Habilidades (CH) & $\begin{array}{l}\text { 1. Dominio de los temas. } \\
\text { 2. Habilidad para usar los diferentes recursos. } \\
\text { 3. Indiferencia ante los temas desarrollados }\end{array}$ \\
\hline Proceso de Aprendizaje (PA) & $\begin{array}{l}\text { 4. Análisis y desarrollo de las actividades } \\
\text { propuestas para la clase. } \\
\text { 5. Incumplimiento en el desarrollo y } \\
\text { presentación de trabajos. } \\
\text { 6. Aplicación de saberes para la solución de } \\
\text { problemas. }\end{array}$ \\
\hline Crecimiento Personal y Social (CPS) & $\begin{array}{l}\text { 7. Cumplimiento de las normas establecidas en el } \\
\text { aula. } \\
\text { 8. Participación en clase. } \\
\text { 9. Uso inadecuado de las redes sociales. } \\
\text { 10. Realización de actividades no académicas (redes }\end{array}$ \\
& sociales, videojuegos, chat, etc.) durante las clases \\
\hline
\end{tabular}

Tabla 5. Escala para establecer el nivel del rendimiento académico

\begin{tabular}{|c|c|}
\hline Rendimiento Académico & Indicadores \\
\hline Bajo & $2,3,5,9,10$ \\
\hline
\end{tabular}

\section{HIPÓTESIS}

Los estudiantes con un promedio menor o igual a 7.0 de la Unidad Académica Preparatoria UAS El Fuerte, Sinaloa, que usan las redes sociales de internet, les afectan significativamente de manera negativa en su rendimiento académico. 
Procedimiento de prueba de hipótesis y correlación de las dimensiones y variables de investigación:

Para acreditar la hipótesis se llevó a cabo mediante la determinación de la correlación entre las variables de investigación mediante el Rho de Spearman aplicado en el SPSS Stadistic v20 determinando el grado o coeficiente de correlación y el nivel de significancia, llevando a cabo el siguiente procedimiento.

Primero, se realizó una agrupación visual en el SPSS Stadistics v20 de los indicadores que permitió realizar la suma de esto para establecer las dimensiones de las variables de investigación, esto se realizó con cada una de las variables determinando el grado o coeficiente de correlación entre los indicadores y cada dimensión de las variables de investigación, así como el nivel de significancia.

Segundo, una vez determinadas las sumas de los indicadores que logro establecer las dimensiones de cada variable, se realizó una correlación determinando el grado o coeficiente de correlación entre las dimensiones y cada variable de investigación, así como el nivel de significancia.

\section{Prueba de Hipótesis}

\section{Tabla 6. Correlaciones}

\begin{tabular}{|c|c|c|c|c|}
\hline & & & $\begin{array}{c}\text { Uso de redes } \\
\text { sociales } \\
\end{array}$ & $\begin{array}{c}\text { Rendimiento } \\
\text { académico }\end{array}$ \\
\hline \multirow{2}{*}{$\begin{array}{l}\text { Rho de } \\
\text { Spearman }\end{array}$} & $\begin{array}{l}\text { Uso de redes } \\
\text { sociales }\end{array}$ & $\begin{array}{l}\text { Coeficiente de correlación } \\
\text { Sig. (bilateral) } \\
\mathrm{N}\end{array}$ & $\begin{array}{c}1,000 \\
\cdot \\
137\end{array}$ & $\begin{array}{c}, 781^{* *} \\
, 000 \\
137\end{array}$ \\
\hline & $\begin{array}{l}\text { Rendimiento } \\
\text { académico }\end{array}$ & $\begin{array}{l}\text { Coeficiente de correlación } \\
\text { Sig. (bilateral) } \\
\text { N }\end{array}$ & $\begin{array}{c}, 781^{* *} \\
, 000 \\
137\end{array}$ & $\begin{array}{c}1,000 \\
\cdot \\
137\end{array}$ \\
\hline
\end{tabular}

**. La correlación es significativa al nivel 0,01 (bilateral).

Dado que el p-valor es menor a 0.05 existe suficiente evidencia estadística para determinar que el uso de las redes sociales está relacionado significativamente con el rendimiento académico de los estudiantes de la unidad académica preparatoria UAS El Fuerte.

Por otra parte, el coeficiente de correlación de Spearman es igual a 0.781 tomando en cuenta que este va de -1 a +1 lo cual indica una relación positiva de nivel fuerte, a mayor uso de las redes sociales menor el rendimiento académico de los alumnos de la unidad 
académica preparatoria El Fuerte.

Tabla 7. Correlación de la dimensión información compartida con la variable uso de redes sociales

\begin{tabular}{|lll|c|c|}
\hline \multicolumn{2}{|c|}{ Correlaciones } & $\begin{array}{c}\text { Uso de redes } \\
\text { sociales }\end{array}$ & $\begin{array}{c}\text { Información } \\
\text { compartida } \\
\text { (agrupado) }\end{array}$ \\
\hline \multirow{2}{*}{ Rho de } & $\begin{array}{l}\text { Uso de redes } \\
\text { sociales }\end{array}$ & $\begin{array}{l}\text { Coeficiente de correlación } \\
\text { Spearman }\end{array}$ & 1,000 &, $512^{* *}$ \\
\cline { 2 - 5 } & $\begin{array}{l}\text { Información } \\
\text { compartida } \\
\text { (agrupado) }\end{array}$ & Coeficiente de correlación &, $512^{* *}$ &, 000 \\
& Sig. (bilateral) &, 000 & 137 \\
\hline
\end{tabular}

**. La correlación es significativa al nivel 0,01 (bilateral).

Dado que el p-valor es menor a 0.05 existe suficiente evidencia estadística para determinar que la dimensión información compartida está relacionado significativamente con la variable uso de las redes sociales.

Por otra parte, el coeficiente de correlación de Spearman es igual a 0. 512 tomando en cuenta que este va de -1 a +1 lo cual indica un nivel de correlación positiva moderada fuerte entre la dimensión información compartida con la variable uso de las redes sociales.

Tabla 8. Correlación de la dimensión beneficios de comunicación con la variable uso de redes sociales.

\begin{tabular}{|c|c|c|c|c|}
\hline & Correla & ciones & Uso de redes & \\
\hline Rho de & $\begin{array}{l}\text { Uso de redes } \\
\text { sociales }\end{array}$ & $\begin{array}{l}\text { Coeficiente de correlación } \\
\text { Sig. (bilateral) } \\
\mathrm{N}\end{array}$ & $\begin{array}{c}1,000 \\
. \\
137 \\
\end{array}$ & $\begin{array}{c}, 534^{* *} \\
, 000 \\
137 \\
\end{array}$ \\
\hline Spearman & $\begin{array}{l}\text { Beneficios } \\
\text { comunicación } \\
\text { (agrupado) }\end{array}$ & $\begin{array}{l}\text { Coeficiente de correlación } \\
\text { Sig. (bilateral) } \\
\text { N }\end{array}$ & $\begin{array}{c}, 534^{* *} \\
, 000 \\
137\end{array}$ & $\begin{array}{c}1,000 \\
\cdot \\
137\end{array}$ \\
\hline
\end{tabular}

Dado que el p-valor es menor a 0.05 existe suficiente evidencia estadística para determinar que la dimensión beneficios de comunicación está relacionado significativamente con la variable uso de las redes sociales. 
Por otra parte, el coeficiente de correlación de Spearman es igual a 0. 534 tomando en cuenta que este va de -1 a +1 lo cual indica un nivel de correlación positiva moderada fuerte entre la dimensión beneficio de comunicación con la variable uso de las redes sociales.

\section{MARCO TEÓRICO}

Desde siempre, el hombre ha tenido la necesidad de comunicarse con los demás, de expresar pensamientos, ideas, emociones, de investigar, saber, obtener información creada, expresada y transmitida por otros. La creación, búsqueda y obtención de información son acciones esenciales y propias a la naturaleza humana, siendo la cultura el fenómeno macro por excelencia de la socialización del conocimiento. Actualmente no hay ninguna dimensión de la vida que quede excluida de la transformación generada por la innovación tecnológica y las posibilidades de manejar la información. (Cornejo \& Tapia, 2011)

Las redes sociales se definen como estructuras en que los diferentes grupos mantienen relaciones sentimentales, amistosas o laborales en el contexto de la web 2.0, multiplicando los espacios de información, discusión e intercambio, de acuerdo con preferencias, intereses, entre otros factores. (MSc. Kelly Deysi Hernández Mite, 2017).

Las tecnologías de las información y comunicación constituye una disciplina encargada del estudio de los medios, materiales, portales web y plataformas tecnológicas al servicio de los procesos de aprendizaje; en cuyo campo se encuentran los recursos aplicados con fines formativos e instruccionales, diseñados originalmente como respuesta a las necesidades e inquietudes de los usuarios (Cañizález, 2017).

La tecnología educativa es un conjunto de recursos, procesos y herramientas de Información y Comunicación aplicadas a la estructura y las actividades del sistema educativo en sus diversos ámbitos y niveles.

Las TIC son el conjunto de tecnologías desarrolladas en la actualidad para una información y comunicación más eficiente, las cuales han modificado tanto la forma de acceder al conocimiento como las relaciones humanas (Chen, 2019).

Más allá de las definiciones puntuales, de lo que semánticamente represente una red social, lo cierto del caso es que ha sido un espacio creado virtualmente para facilitar la interacción entre personas. Desde luego, esta interacción está marcada por algunos aspectos particulares como el anonimato total o parcial, si así el usuario lo deseara, la 
facilidad de contacto sincrónico o anacrónico, así como también la seguridad e inseguridad que dan las relaciones que se suscitan por esta vía. (Hütt Herrera, 2012)

Por lo que el aprendizaje de los alumnos implica saber escuchar, interpretar y emitir los mensajes pertinentes en diversos escenarios haciendo uso de los medios, códigos y herramientas apropiadas.

En este contexto, si al inicio pudieron ser los correos electrónicos o el chat, pronto el uso del blog y los sitios de acceso libre constituyeron una valiosa herramienta para que los docentes mantuvieran comunicación con sus alumnos y colegas académicos, lo que ha permitido la constitución de efectivas comunidades virtuales, detrás de lo cual se esconde un cambio de paradigma en las prácticas docentes (Aguirre Aguilar, 2012).

El 19 de febrero de 2014, la aplicación fue comprada por la empresa Facebook por 19000 millones de dólares (de los cuales 12000 millones corresponden a acciones de Facebook y el resto en efectivo). A principios de octubre se anuncia la compra definitiva de WhatsApp por Facebook por valor de 21800 millones de dólares. Algunas semanas después de la compra, WhatsApp anunció tener capacidad para realizar video llamadas en el verano del mismo año. El despliegue definitivo del Voip llegó progresivamente durante 2015 a todas las plataformas móviles.

Google Inc. es la empresa propietaria de la marca Google. Fue fundada el 4 de septiembre de 1988, en Menlo Park, California, por Larry Page y Sergey Brin, entonces estudiantes del doctorado en ciencias de la computación en la Universidad de Stanford, Estados Unidos y quienes se conocieron en 1995. Page y Brin desarrollaron una fórmula matemática que clasificaba una página web según la cantidad de otras páginas web vinculadas a ella. El nombre de la marca -explica Thomas Friedman (1996), autor del libro La Tierra es plana. Breve historia del mundo globalizado del siglo XXI. (Islas, 2009) A diferencia de Microsoft -que no está dispuesta a compartir los códigos de sus programas-, Google ha conseguido establecer una positiva imagen entre programadores independientes por liberar los códigos de sus aplicaciones. La comunidad internacional de desarrolladores de software ha procedido a mejorar y reconstruir las aplicaciones que Google libera, situación que estimula el desarrollo de "mash ups" -remezclas de aplicaciones-". Evidentemente se ha desarrollado una gran rivalidad entre ambas marcas. La OPA de Microsoft sobre Yahoo precisamente es expresión de la agresiva competencia que prevalece entre Google y Microsoft. 
Actualmente las tecnologías siguen innovando e impactando de forma acelerada y sorprendiendo cada vez más con sus múltiples funciones al ámbito educativo (UNAM, 2015).

El desarrollo impetuoso de las redes sociales ha permitido la globalización de la información, mejorando las relaciones sociales, políticas, económica religiosas etc., a una escala inimaginable hace década y media.

Si hablamos de competencias en general, nos referimos al conjunto de conocimientos, habilidades prácticas, actitudes, motivaciones, emociones, etc. que se unen para completar una acción de forma eficaz. Cuando nos referimos a la Competencia Digital Docente, aplicamos esta definición en la relación que tienen los docentes con el uso de las Tecnologías de la Información y Comunicación en su práctica educativa (Marquez, 2017).

\section{Las redes sociales}

Desde que surgieron, en 2005, han cobrado fuerza como medio de intercambio de ideas y contenido. Une varios servicios, como mensajería privada, chat, publicación de mensajes, foros, y páginas. También se ubica en los recursos de la Web 2.0. Entre los más mencionados están:

Twitter

$\checkmark$ Facebook

$\checkmark$ MySpace

\section{Hacer uso de las redes sociales permitirá al docente:}

- Fomentar el trabajo colaborativo en su asignatura.

- Abrir la asignatura más allá de las paredes del aula.

- Acceder al conocimiento al instante y seguir actualizado.

- Crear una red de contactos docentes y profesionales, a la vez que acerca a los alumnos de su asignatura a la realidad profesional.

- Desarrollar competencias informacionales que permitan gestionar la gran cantidad de información que circula en la red.

- Crear o mejorar una reputación y visibilidad en la red.

- Compartir o reflexionar los trabajos generados y experiencias.

Estas tecnologías permiten a los alumnos interactuar con los contenidos y comunicarse a través de diferentes medios, seleccionarlos, remezclarlos, crearlos y compartirlos. 
De forma que más que el interés por el uso de las herramientas que nos proporcionan las redes sociales, hay que poner de relevancia su indudable utilidad como medio para fomentar el contacto, el diálogo y la comunicación entre alumnos y profesores. Especialmente como favorecedor de la comunicación en su vertiente docente de enseñanza y aprendizaje, sin despreciar la personal que como en cualquier red social no educativa se puede ver potenciada.

Las redes sociales como el Facebook, realmente llaman la atención de los jóvenes, estas herramientas pueden llegar a contribuir de manera significativa en todos los aspectos de su formación (Nájera \& Meza De la Hoz, 2015)

Estudios revelan que Facebook es un sitio web que permite alojar un sinnúmero de caras (facetoface), contextualizando diversos estilos de vida, teniendo acceso a información de tipo confidencial, la cual puede ser utilizada con fines delictivos o lucrativos; por ello, es recomendable concientizar el tipo de información difundida en cada una de las publicaciones o perfiles emitidas principalmente por los adolescentes.

Es fácil aprovecharlo como centro de comunicaciones e intercambio de ideas. Pero sobre todo la importancia de familiarizarse y entender una red social en la que tus estudiantes se sienten muy cómodos pero necesitan modelos de cómo usarlo con seguridad y responsabilidad.

Twitter o microblogging; Es una red social que cobró fuerza en 2006. Al igual que Facebook, permite tener varios servicios en un mismo sitio: mensajería, chat, foros, sitios. En el ámbito educativo, permite generar comunidades de aprendizaje entre estudiantes y docentes. Se abre un canal de comunicación en donde los alumnos pueden estar informados sistemáticamente mediante conversaciones diarias, intercambio de información y reportes de noticias o avisos. A partir de una contextualización de cómo debiera darse la apropiación de las tic en la práctica docente y un recuento de las principales herramientas usadas actualmente, se expone una experiencia de aprendizaje docente en la Universidad Veracruzana (Genaro \& Rocio, 2012).

El rendimiento académico va unido a la calidad y a la eficiencia del sistema y, de hecho, el rendimiento educativo se presenta, normalmente, como un índice para valorar la calidad global del sistema (Quiñones, 2007).

El elemento clave para la integración de las redes sociales en el aula sigue siendo el profesorado, sin su implicación no se llevará a cabo este proceso. 
Así mismo Navarro (2003) menciona, que la complejidad del rendimiento académico inicia desde su conceptualización, en ocasiones se le denomina como aptitud escolar, desempeño académico o rendimiento escolar, pero generalmente las diferencias de concepto sólo se explican por cuestiones semánticas, ya que generalmente, en los textos la vida escolar y la experiencia docente, son utilizadas como sinónimo.

La integración de las TIC en educación es un proceso complejo en el que intervienen muchos factores (Cerveró, 2011).

La virtualización de la educación ha dado paso a una serie de críticas sobre su eficiencia y eficacia. Las críticas giran alrededor de las experiencias de aprendizajes virtuales y cuestionan su valor en la generación de aprendizajes significativos y permanentes. Otro aspecto controversial hace referencia a la calidad académica, ya que involucra un cambio de paradigmas pedagógicos y didácticos en la presentación y adquisición de la información, en las competencias requeridas tanto para el docente y para el estudiante, en el cambio de roles, entre otros (Méndez, 2012).

En ningún caso el docente debe convertirse en un controlador o policía de lo que hacen sus estudiantes en el aula. Su función es coordinar y facilitar el aprendizaje y la mejora de la calidad de vida del alumnado.

El conocimiento está en la red y es abundante, pero precisamente esto es lo que hace necesario un buen número de tareas que debe cumplir todo docente: detectar lo realmente importante, guiar los procesos de búsqueda, analizar la información encontrada, seleccionar la que realmente se necesita, interpretar los datos, sintetizar el contenido y difundirlo son algunas de las tantas tareas que el profesor debe guiar (Amigo, 2016).

El rendimiento escolar es un "nivel de conocimientos demostrado en un área ó materia comparado con la norma de edad y nivel académico", encontramos que el rendimiento del alumno debería ser entendido a partir de sus procesos de evaluación, sin embargo. (Edel Navarro, 2003)

\section{ANALISIS DE RESULTADOS Y DISCUSIÓN}

Una red social es una estructura social de la que forman parte varias personas $\mathrm{u}$ organizaciones. Por ejemplo, podría ser un grupo de amigos o una familia, porque son personas que comparten vínculos y que se comunican entre ellas. Es un concepto que se utiliza en sociología, la ciencia que se encarga de estudiar la sociedad humana. (Ros, 2020) 
Es necesario hacer las siguientes interpretaciones de acuerdo a los resultados obtenidos del instrumentos consistente en encuesta dirigida a los alumnos con un promedio menor o igual a 7.0 de la Unidad Académica Preparatoria El Fuerte, de la Universidad Autónoma de Sinaloa, que nos lleva a conocer tiempo, la frecuencia, el fin y lo que hace el estudiante con las redes sociales; analizando estos indicadores, nos arroja que el 95.6\% de los encuestados conocen que es una red social (Figura 5 ), de los cuales el $83.2 \%$ es miembro de una red en internet (Figura 6 ) siendo el caso de que el $87.6 \%$ cuenta con un perfil de Facebook (Figura 7).

De acuerdo a lo que dice Tamayo (2017), Facebook es una de las redes sociales más populares en todo el mundo. Con millones de usuarios conectados entre sí, ofrece grandes oportunidades de comunión a quienes forman parte de esta red.

Atendiendo los resultados de la Figura 12 consistente en lo que el alumno hace en las redes sociales de internet el $18.98 \%$ comparte actividades académicas y en igual porcentaje comparte fotos y videos. Encontramos que el 3.65\% establece que la red social la utiliza para la creación de nuevos contactos, así como el $45.26 \%$ lo utiliza para chatear. Como se observa en los resultados interpretados en el párrafo anterior el porcentaje que corresponde al uso para fines académicos de una red social a la que es miembro el alumno, es relativamente muy inferior a la que corresponde al uso para chatear, así como también, en el mismo porcentaje en el que el alumno realiza actividades académicas lo hace para compartir videos y fotografías, así pues sumando el porcentaje que corresponde al uso para el chat y para compartir videos y fotografías nos arroja que el $64.24 \%$ utiliza las redes sociales con fines de entretenimiento.

\section{Figura 1}

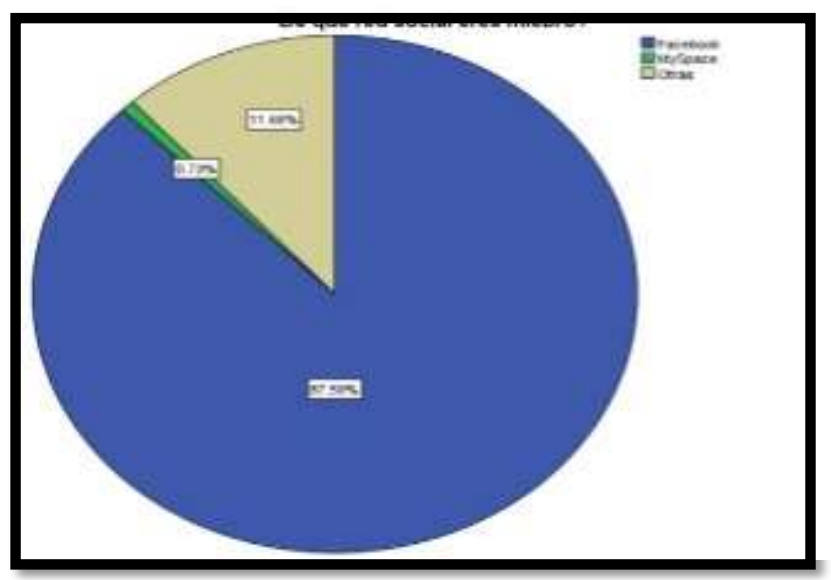

Figura 1. ¿De qué red social eres miembro? 


\section{Figura 2}

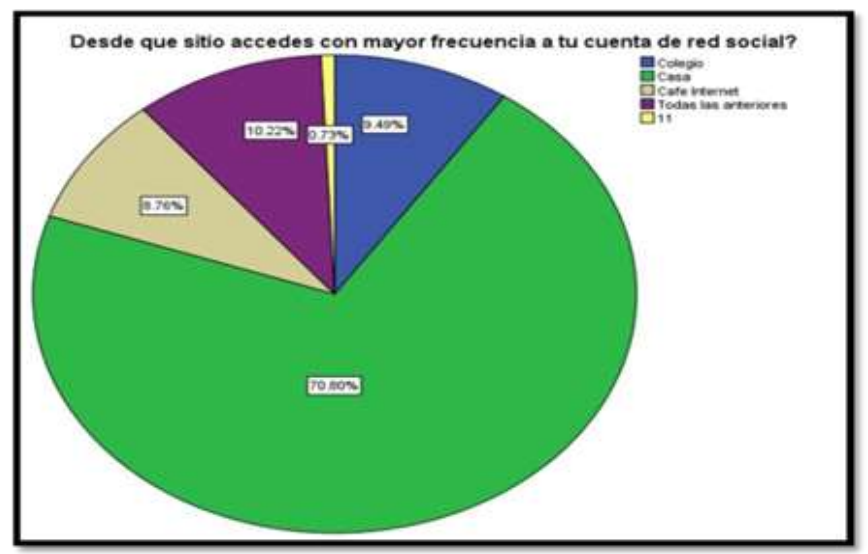

Figura 2. ¿Desde qué sitio accedes con mayor frecuencia a tu cuenta de red social?

Figura 3

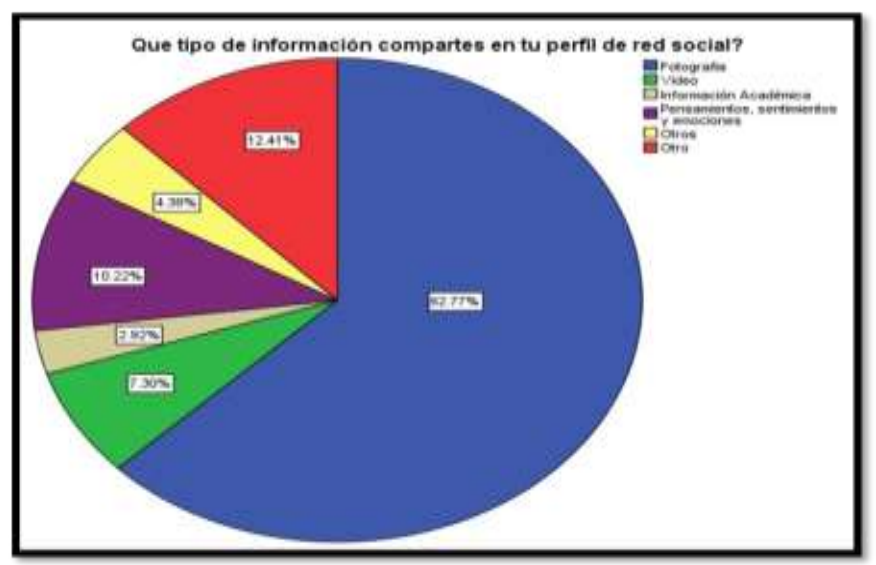

Figura 3. ¿Qué tipo de información compartes en tu perfil de red social?

\section{CONCLUSIONES}

Se identifica que más del $50 \%$ de los estudiantes tienen acceso a internet desde una computadora en su hogar; se logra conocer que los alumno que fueron objeto de estudio manifiestan en casi su totalidad saber que es una red social, además que también se reflejó que más de las tres cuartas partes son usuarios de una red social, que usan frecuentemente, independientemente del sitio que lo hagan.

En relación a la frecuencia y tiempo de uso de las redes sociales, los alumnos acceden en su mayoría una vez o más de una vez al día, puede ser por menos de una hora a 4 horas, sin embargo, es considerable e impactante que también usen una red social de manera 
excesiva pues hay quienes lo hacen por 5 horas o más. El riesgo más importante del abuso de las redes sociales puede generar una adicción, dentro de las cuales como señal de alarma podemos identificar tiempos de conexión anormalmente altos, descuidar otras actividades importantes, como el contacto con la familia, las relaciones sociales, el cuidado de la salud y su rendimiento académico.

De acuerdo a la observación participante, como instrumento de recolección de información, donde el autor es miembro activo en la institución objeto de estudio, se determina que los estudiante cuando acceden a internet con miras de cumplir con tareas escolares, solamente dedican un periodo de tiempo relativamente corto ya que la mayoría del tiempo lo dedican para buscar información que nada tiene que ver con los contenidos escolares, si no con información o contenidos que satisfagan su libido, su morbo y su entretenimiento lo cual no abonan al rendimiento académico de los jóvenes estudiantes de la preparatoria El Fuerte.

Según los resultados obtenidos del análisis de información, la mayoría de los estudiantes son miembros de una red social, utilizan en su gran mayoría Facebook, en la que comparten fotografías, videos, pensamientos, sentimientos y emociones, buscan nuevos contactos y utilizan el chat principalmente, evidentemente se manifiesta que están utilizando las redes sociales para su entretenimiento.

En base a la experiencia y en relación al uso de las redes sociales y el internet, los estudiantes del bachillerato de la UAS específicamente la escuela preparatoria de El Fuerte lo utilizan para satisfacer necesidad de intercomunicación con los demás, al igual que comparten espacios virtuales, buscando irrumpir en los espacios de los demás, en pocas ocasiones utilizan estos espacios para realizar tareas escolares que sirvan para su formación académica.

Lo expresado con antelación me permite tener un diagnóstico con el cual podemos afirmar que los encuestados acceden de alguna u otra manera, desde su casa o cualquier otro lugar a internet con el fin de acceder a la red social de su preferencia, determinándose que lo hacen una vez al día o más de una vez al día dedicándole un periodo de tiempo que oscila entre dos y tres horas a día, así mismo se afirma que valoran el uso de las redes sociales de internet cuando manifiestan que las aprovechan para beneficio académico. 
Casi las dos terceras partes de los alumnos encuestados manifiestan no afectarles en su rendimiento académico el uso de las redes sociales de internet, ya que investigan, buscan información y desarrollan tareas, según su opinión su uso los beneficia académicamente. Partiendo de los datos que no arroja la encuesta pudimos conocer que los estudiantes se sienten motivados en el uso de las redes sociales, ya que consideran que les trae un beneficio académico; la gran mayoría manifiesta acceder a una red social para hacer tareas, investigar y buscar información, realizan actividades, como compartir fotografías, videos pensamientos, sentimientos, emociones y con mayor frecuencia para chatear, en su mayoría usan las redes sociales para entretenimiento.

Con esto podemos afirmar que distribuyendo el tiempo de acceso y todas estas actividades se destina un tiempo muy breve para la realización de actividades académicas, ya que la mayoría accede una hora o meno al día, si consideramos la tabla número 12 y la figura 12.1, podemos corroborar que existe un nulo interés por actividades académicas cuando accede a una red social de internet y cuando lo hacen, más de las dos terceras partes algunas veces postergan las actividades académicas.

De acuerdo al análisis de resultados el tiempo que le dedica siempre esta compartido con otras actividades y preferentemente lo utiliza para la realización de actividades de entretenimiento.

En relación al seguimiento de datos en la búsqueda de cumplir con el objetivo general de investigación que es determinar la influencia que ejerce el uso de las redes sociales de internet en el rendimiento académico de los estudiantes con promedio menor o igual a 7.0 de la unidad académica preparatoria UAS El Fuerte, Sinaloa; podemos afirmar que la mayoría de los alumnos encuestados no utilizan las redes sociales para realizar actividades académicas, la otra mitad acceden a internet en búsqueda de tareas y para investigar, una reducida cantidad de ellos, destaca que la usan una vez con más frecuencia quedando un número muy reducido de los que acceden más de tres veces, con esto se determina que los estudiantes objeto de estudio utilizan las redes sociales frecuentemente con fines que no tienen nada que ver con sus actividades académicas, se encontró que el estudiante cuando usa las redes sociales de internet, en muy pocas ocasiones, está manejando información que pueda abonar a su rendimiento académico.

El uso de las redes sociales se ha vuelto una adicción en algunas de las personas, ya que es tanta su obsesión por el uso del internet que han puesto en peligro tanto sus estudios 
como sus relaciones personales. Dentro de esto se debe tomar en cuenta que la mayoría de los jóvenes pasan más tiempo atendiendo el móvil y leyendo publicaciones que nada tiene que ver con su rendimiento académico o que influyen en sus actividades escolares. Lo anterior nos llevó a la contratación de la hipótesis que consistió en que los estudiantes con un promedio menor o igual a 7.0 de la Unidad Académica Preparatoria UAS El Fuerte, Sinaloa, que usan las redes sociales de internet, les afectan significativamente de manera negativa en su rendimiento académico, en virtud de que las dimensiones que componen cada una de las variables se relacionan significativamente en un grado o coeficiente de correlación positiva fuerte, lo que permitió que mediante el Rho de Spearman, comprobar cómo verdadera la hipótesis de la investigación ya que el grado en mención, indica que el uso de las redes sociales de acuerdo a los criterios y dimensiones establecidas impacta negativamente en el rendimiento académico de los estudiantes de la Unidad Académica Preparatoria El Fuerte de la UAS.

\section{BIBLIOGRAFÍA}

Aguirre Aguilar, R. M. (2012). Competencias digitales y docencia: una experiencia desde la práctica universitaria. Innovación Educativa, 1-12.

Amigo, V. B. (2016). El rol del docente en la era digital. Revista Interuniversitaria de Formación del Profesorado, 103-114.

Callejo Gallego, J. (2002). OBSERVACIÓN, ENTREVISTA Y GRUPO DE DISCUSIÓN: EL SILENCIO DE TRES PRÁCTICAS. Revista Española de Salud Pública, 409-422.

Cañizález, T. (2017). Tecnología educativa y su papel en el logro de los fines de la educación. Educere, 1-9.

Cerveró, A. (2011). Las competencias y el uso de las Tecnologías de Información y Comunicación (TIC) por el profesorado: estructura dimensional. REDIE. Revista Electrónica de Investigación Educativa, 28-42.

Chen. (21 de 05 de 2019). Significado de TIC (Tecnologías de la información y la comunicación). Recuperado el 28 de 07 de 2021, de Significado de TIC (Tecnologías de la información y la comunicación): https://www.significados.com/tic/

Cornejo, M., \& Tapia, M. L. (2011). Redes sociales y relaciones interpersonales en internet. Fundamentos en humanidades, 219-229. 
Edel Navarro, R. (2003). El rendimiento académico: concepto, investigación y desarrollo. REICE. Revista Iberoamericana sobre Calidad, Eficacia y Cambio en Educación, 3-16.

Genaro, A., \& Rocio, R. M. (2012). Innovacion educativa (Mexico D.F). Scielo, 1-59.

Hütt Herrera, H. (2012). LAS REDES SOCIALES: UNA NUEVA HERRAMIENTA DE DIFUSIÓN. Reflexiones, 121-128.

Islas, O. (2009). GOOGLE. LA MARCA EMBLEMÁTICA DE INTERNET 2.0. Razon y palabra, 8-16.

Malinowski, B. (1967). Una teoría científica de la cultura. Buenos Aires: Edhasa.

Marquez. (02 de 10 de 2017). ¿Que nivel de competencia digital docente deberiian tener los profesionales de la enseñanza? Recuperado el 28 de 07 de 2021, de ¿Que nivel de competencia digital docente deberiian tener los profesionales de la enseñanza?: https://yosoytuprofe.20minutos.es/2017/10/02/que-nivel-de-competencia-digitaldocente-deberian-tener-los-profesionales-de-la-ensenanza/

Méndez, G. A. (2012). Competencias digitales y docencia: una experiencia desde la práctica universitaria. Innovación educativa , 1-16.

MSc. Kelly Deysi Hernández Mite, M. J. (2017). LAS REDES SOCIALES Y ADOLESCENCIAS. REPERCUSIÓN EN LA ACTIVIDAD FÍSICA. Revista Universidad y Sociedad, 30-42.

Nájera, F. S., \& Meza De la Hoz, L. (2015). Facebook como herramienta educativa en el proceso de enseñanza-aprendizaje en el nivel medio superior. Zona Proxima, 116127.

Quiñones, S. H. (2007). Rendimiento Escolar. Humanidades, Tecnologia y Ciencia, 1-5. Ros, L. (29 de 06 de 2020). La Vnaguardia. Recuperado el 02 de 09 de 2021, de La vanguardia: https://www.lavanguardia.com/vida/juniorreport/20200629/482009621616/dia-redes-sociales.html

Sampieri, R. H. (2014). Metodologia de la Investigación. México: Mc Graw Hill.

UNAM. (01 de 09 de 2015). rdu revista digital universitaria. Recuperado el 03 de 06 de 2021, de rdu revista digital universitaria: http://www.revista.unam.mx/vol.16/num9/art76/

\section{SINTESIS CURRICULAR}




\section{Rosalba Trinidad Chávez Moreno}

Dra. en Ciencias Sociales, por la Universidad Autónoma Intercultural de Sinaloa, Profesora de Tiempo Completo en la Universidad Autónoma de Occidente, Unidad Regional El Fuerte, Teléfono: 6683961800. Correo electrónico: rochamor2@ gmail.com

\section{Francisco Antonio Romero Leyva}

Dr. Educación y diversidad, por la Escuela Normal de Especialización del Estado de Sinaloa, Profesor Tiempo Completo de la Universidad Autónoma Indígena de México, Teléfono 6683204435, Correo electrónico roleyva131@ @otmail.com

\section{Teodoro Lugo Tapia}

Dr. en Ciencias Sociales, por la Universidad Autónoma Intercultural de Sinaloa, Profesor de Medio Tiempo en la Universidad Autónoma de Occidente, Unidad Regional El Fuerte, Teléfono: 6981096606. Correo electrónico: $\underline{\text { lugot63@hotmail.com }}$

\section{Apodaca López Carlos Alberto López}

Pasante de Maestría en Docencia por la Universidad Autónoma de Occidente, Profesor supernumerario en la Universidad Autónoma de Occidente, Unidad Regional El Fuerte, Teléfono: 6981062799. Correo electrónico: carlosp175@live.com.mx

\section{Félix Ortiz Georgina}

Dra. en Desarrollo Humano y Educación, por el Centro Universitario Mar de Cortes, Profesora de Tiempo Completo en la Universidad Autónoma de Occidente, Unidad Regional Los Mochis, Teléfono: 6682650616. Correo electrónico: gfelixortiz@gmail.com

\section{Silvia Ortiz Castro}

Dra. en Innovación y administración Educativa, por el Centro Universitario de Ciencias e Investigación, Profesora de Tiempo Completo en la Universidad Autónoma de Occidente, Unidad Regional Los Mochis, Teléfono: 6681623572 Correo electrónico: Silvia.ortiz@uadeo.mx 